\title{
Promoting innovation in healthcare
}

\author{
Authors: Christopher J Kelly ${ }^{\mathrm{A}}$ and Antony J Young ${ }^{\mathrm{B}}$
}

Innovation can be defined as invention + adoption + diffusion. In healthcare, it may be a novel idea, product, service or care pathway that has clear benefits when compared to what is currently done. Successful innovations often possess two key qualities: they are both usable and desirable. How can proven innovations be quickly and effectively adopted as best practice and taken up across the whole healthcare system? How can the commercial success of our ideas be realised at home rather than abroad, as has too often been the case? This review explores important issues of funding, information governance, interoperability, medical device regulation, procurement, clinical prototyping and the systemic challenge of encouraging and scaling innovation.

KEYWORDS: Healthcare, innovation

\section{Introduction}

Innovation can be defined as invention + adoption + diffusion. In healthcare, it may be a novel idea, product, service or care pathway that has clear benefits when compared to what is currently done. Successful innovations often possess two key qualities: they are both usable and desirable.

Historically, the NHS and our academic partners have led the world in inventing and testing potential innovations. However, challenges remain. How can proven innovations be quickly and effectively adopted as best practice and taken up across the whole healthcare system? How can the commercial success of our ideas be realised at home rather than abroad, as has too often been the case? The situation has to change if we want our patients to receive the first-hand benefits of innovation.

Healthcare is not unique in slow adoption and diffusion. It took the telephone 64 years, electricity 45 years, computers 23 years, mobile phones 16 years, radio 12 years and the internet 13 years to achieve $40 \%$ consumer adoption. ${ }^{1}$ But other sectors have made progress in innovating better, faster

Authors: ${ }^{\mathrm{A}} \mathrm{NHS}$ England clinical entrepreneur and clinical research fellow, Centre for the Developing Brain, St Thomas' Hospital, London, UK; ${ }^{B}$ consultant urological surgeon, Southend University Hospital; director of medical innovation, Anglia Ruskin University; innovation lead, Mid and South Essex STP; national clinical lead for innovation, NHS England and smarter than in healthcare. Successful companies ask their customers what they want and innovate based on their findings. They identify the key pain points and work with customers, other stakeholders and experts to solve the issue, develop the strategy for adoption and diffusion, and then deliver it. Such organisations have developed and provided a culture of innovation for their employees.

\section{The challenge of innovating in healthcare}

The NHS faces a number of key issues that need to be addressed if we are to successfully innovate:

1 budgets are limited - the NHS has to make efficiency

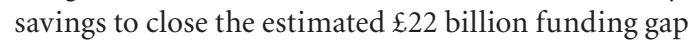
between patients' needs and resources ${ }^{2}$

2 demand is increasing - our population is living longer than ever before, often with multiple comorbidities

3 the front line is under increasing pressure - as clinical demands increase, new ways of doing things are often the first casualty

4 public expectations are rising

5 accepting failure as part of the innovation process - there is no penalty for following custom and practice, but if you try and make an innovative change and it fails, the repercussions for the clinician or the trust/clinical commissioning group board can be considerable.

\section{Creating a culture for innovation}

Many NHS staff have great ideas for improving the system and patient experience. If we are to realise the benefits from this, we must create a culture where innovation can flourish, and where our staff and patients feel empowered to contribute to the innovation process and help make change happen. Many of the building blocks that form the foundation of a more innovative culture are already in place. ${ }^{3}$ Some of the key culture-building components are listed in Box 1.

\section{Accessing funding for innovation}

For new innovations to flourish and spread at scale, access to adequate funding is critical. There is an increasing range of both public and private funding sources in the UK although public funds remain limited and awareness among NHS staff of what is available could be improved.

The healthcare-focused angel and venture capital sectors continue to mature in the UK, while the government's enterprise investment scheme tax relief for investors in start-up 
Box 1. Key NHS offerings that contribute to a culture supporting innovation

> Best practice tariffs - incentivise and reimburse providers for the cost of high-quality, innovative care

> Innovation Challenge Prizes - a national level celebration and reward for outstanding innovation in healthcare

$>$ Healthcare Small Business Research Initiative - precommercial procurement contracts for research and development in innovative healthcare products and services

Clinical excellence awards - rewarding clinicians for quality improvement, research and innovation

> Academic Health Science Networks - 15 regional organisations bringing together healthcare, academia and industry on an open innovation platform to lead work in the NHS on adoption and diffusion of innovation

The National Institute for Health and Care Excellence (NICE) - including the NICE Implementation Collaborative, Medical Innovation Briefings, Medical Technologies Evaluation Programme, NICE reviews and guidelines

$>$ NHS England Innovation portal - innovation connect and exchange provides support, advice and navigation through the system and is open to all who want to innovate and collaborate in healthcare

> Innovation Scorecard - enables benchmarking and increases transparency for the variation of adoption in NICE technology appraisals

> 'Test beds' - collaborations between the NHS and innovators that harness technology to address some of the most complex issues facing the health service. They provide real world sites testing combinatorial innovations that can deliver better care, better patient experience of care at the same or lower overall cost

> National Innovation Accelerator - a fellowship programme equipping innovators with the skills knowledge and experience to drive the uptake of their proven innovations across the NHS.

$>$ Health and Social Care Innovation Expo

Healthy New Towns programme - improving health through the built environment, reshaping the health of communities and rethinking how health and care services can be delivered.

A Continuing Clinical Innovation (CCI) points scheme - this will become part of the continuing professional development scheme. A framework will be provided that allows clinicians and their appraisers to award CCI points for innovative activity that will count towards their annual appraisal and revalidation

> The Innovation Technology Tariff - national level reimbursement for new medtech innovations ${ }^{4}$

> The Clinical Entrepreneur Programme - a workforce development programme allowing clinicians to continue their frontline NHS roles while developing their entrepreneurial ideas companies continues to attract capital and talent from around the world. Technologies coming out of the leading universities and academic centres in the UK have enabled the success of deep technology accelerator programmes like Entrepreneur First, helping take new technologies from academic concept to a viable commercial enterprise.

The popular NHS Challenge Prizes and local hospital innovation prizes offer limited initial funding to develop new ideas. Further funding has been facilitated by the Academic Health Sciences Networks, particularly through the National Innovation Accelerator focused at individuals working on an innovative product, and the Small Business Research Initiative that provides funds to small businesses to develop innovative products that address unmet health needs. In addition, the NHS chief executive Simon Stevens recently announced a new funding route (the 'Innovation and Technology Tariff'), which will provide an explicit national reimbursement route for new medtech innovations, including apps, and enable their uptake across the NHS at scale. ${ }^{4}$ The National Institute for Health Research has a number of funding schemes that support medical innovation. One of the most popular of these has been the Invention for Innovation Programme, which allows earlystage funding for clinicians to work with both academics and companies to develop their ideas. ${ }^{5}$

\section{Addressing some key issues}

There are a number of areas that present barriers to innovation in healthcare. We need to tackle these if we are to make innovation happen.

\section{While privacy is very important, so too is data sharing}

The handling of confidential patient data is a fundamental requirement for many potential innovations in the NHS, and this has historically been one of the greatest hurdles to getting new products to market. Given the potential for harm, many NHS organisations have isolated themselves from the outside world with all-encompassing firewalls to protect systems from attack. This approach has arguably been to the detriment of innovations that could have been built upon the digital health record.

Information governance processes can appear a frustrating barrier to anyone who wants to improve how their NHS service is organised and delivered, with many feeling that information governance has become overly-complex, too risk averse and too inflexible to meet the modern needs of patients and clinicians. NHS England has acknowledged the opportunity for improved processes and pledged to simplify information governance processes and tools, work towards providing a consistent approach to information governance and remove duplication of assessment work. ${ }^{6}$ Data sharing is essential to delivering integrated care, improving patient experience and conducting research - achieving a pragmatic approach to information governance will be vital.

\section{Interoperability is critical to improving healthcare quality, safety and efficiency}

The past decade has seen a dramatic shift from largely paperbased health records to most GP surgeries and a growing 
number of trusts in the UK using electronic health records. However, the task of digitising the NHS health record has been a long, complex and, at times, challenging process, ${ }^{7}$ resulting in the purchase of a huge variety of different systems with consequent interoperability issues. The deadline for a paperless NHS started off as $2018,{ }^{8}$ then $2020^{9}$ and the recent Wachter report now recommends aiming for 2023..$^{10}$

Digitising the record is just the first step towards achieving healthcare improvement. Benefits can only be truly realised once all the individual systems and databases have been connected, including enterprise systems, third party apps and patient-facing systems. At this point, we can start gleaning valuable insights from the data, and converting these insights into actions that will improve quality, safety and efficiency. In the UK, we are currently addressing the challenges of achieving both digitisation and interoperability. By implementing the recommendations of the Wachter report, we will enable an interconnected, mixed ecosystem of IT system providers, and build the essential foundations for the health innovations of the future.

\section{Intellectual property policy should not stifle new innovations}

Delivering fair and rational intellectual property treatment to innovators within the NHS is a vital ingredient to promoting innovation in the NHS. Battles around intellectual property can result in great ideas being stifled, with the result that their potential is never realised. This is clearly not in the interest of the NHS or our patients. There are multiple legal areas that need to be considered, including the Patents Act, employment contracts (agreed both nationally and locally) and NHS England/clinical commissioning group/provider contracts. The Department of Health last published a framework and guidance on the management of intellectual property in the NHS in $2001,{ }^{11}$ a time before the mainstream adoption of smartphones and when the internet was in its relative infancy.

We need to create an environment where the optimal place to develop an idea is within the NHS. In combination with access to funding, support and appropriate guidance, this goal can be achieved.

\section{Education about app development and medical device regulation}

Many great ideas fall at the barrier of regulation. Until recently, the ambiguity of how medical device regulation should be applied to medical apps has proved a challenge to many app developers across the NHS. The Medicines and Healthcare products Regulatory Agency (MHRA) published welcome guidance in late 2016, which explained the assessment process in much greater detail. ${ }^{12}$ Outside of the MHRA, the US Food and Drug Administration has described a specific subset of apps that it wishes to regulate (those used as an accessory for a regulated medical device, or that transform the mobile platform into a regulated medical device), while outlining a wider range of apps over which it intends to exercise enforcement discretion. ${ }^{13}$ To assist the many healthcare professionals interested in building new apps for use in their work, it may be beneficial to produce an accessible, concise and practical guide to developing medical apps that meet regulatory standards.
This could help future innovators ensure that their apps are appropriately quality-assured for use in the NHS.

\section{Procurement is a significant hurdle for small businesses}

The NHS procurement process can appear baffling, complicated and unnecessarily bureaucratic. The coalition government recognised this problem in 2012 and the Department of Health subsequently published an action plan to support small and medium-sized enterprises selling to the NHS. ${ }^{14}$ The central theme was increasing transparency and engagement with small businesses, with a goal to double Department of Health spend with small and medium-sized enterprises over the subsequent 2 years. The fifteen Academic Health Sciences Networks across the UK have helped facilitate this mission, connecting small businesses with the many components of the wider NHS, enabling innovation at greater scale and ease than was possible before.

\section{Prototyping and testing innovations in a clinical environment}

All devices, services and pathways require evidence to support their introduction, and must show superiority to what is currently available, ideally at a lower cost. However, this evidence is difficult to acquire without access to patients or clinical facilities to develop and test prototypes. As the world's leading fully integrated health system, the NHS represents an exciting opportunity for innovators. In January 2016, the NHS announced the creation of seven 'test beds' to partner global innovators with NHS organisations to evaluate the real-world impact of new technologies. ${ }^{15}$ The 'test beds' cover different aspects of NHS care, from managing long-term conditions to exploring health applications of the Internet of Things.

Another important development was the creation of the new care model vanguards programme in 2015. This brings together partnerships of NHS organisations, local government, voluntary, community and other organisations in order to move from fragmented care to integrated care in communities. There is already evidence that vanguards are improving local services, while reducing pressure on busy GP and accident and emergency services. ${ }^{16}$ The Department of Health announced $\mathfrak{E} 101$ million of new funding in December 2016 to support and spread this important work. Such incentives offer valuable opportunities for companies across the world to develop innovation at scale in the UK.

\section{The systemic challenge of encouraging and scaling innovation}

There are many examples of promising innovation in the NHS, but very few that have been able to scale beyond their single local area or site. Innovations that specifically address one region's local needs are not necessarily a problem, but if they are not joined up across the system they can result in negative consequences. Firstly, additional energy must be expended in other areas to address the same problem. Secondly, many highly localised solutions result in an overly complex organisation without common points for integration. If we 'let a thousand flowers bloom', we may end up with a field of weeds. However, focusing down too soon may prevent the innovations we need 
Box 2. Case study

NeoMate is a smartphone app designed by junior paediatric doctor, and now NHS England clinical entrepreneur fellow, Christopher ] Kelly. It aims to help doctors and nurses looking after sick newborn babies by offering drug and infusion calculations, intubation and central line calculations, checklists for common acute situations and concise reference material.

The app was originally designed by me for me, during my first paediatric job, after experiencing the realities of a stressful neonatal resuscitation in a small district general hospital - says Kelly.

The challenge in releasing the app began when Apple refused to publish a drug-calculating app written by an individual. Kelly partnered with the London Neonatal Transfer Service, an intensive care ambulance service that transports sick babies between hospitals, which adopted the app and helped introduce a rigorous governance process to ensure quality and accuracy. A small team of doctors, nurses and paediatric pharmacists carefully checked and helped improve the app over several months and worked to ensure that the app met requirements for CE marking.

The final organisational challenges that we experienced in publishing the app were greatly helped by winning an NHS Challenge Prize in 2015 - says Kelly. This gave everyone the additional confidence to officially launch the app on iPhone and Android.

Funding from the NHS Challenge Prize provided resources to promote and develop the app further within the NHS and abroad, with the app since being downloaded almost 60,000 times across 195 countries.

Being part of the NHS Clinical Entrepreneur Programme has allowed me to meet a wide range of interesting people, learn new skills and knowledge, and gain a unique insight into the world of medical innovation. There's a special atmosphere of energy and enthusiasm at each event as part of the programme, which is unique in the NHS at the moment.

from being developed. This is the innovation dilemma faced in healthcare. As projects mature, it can remain difficult to distinguish flowers from weeds unless care is taken to innovate in a focused, managed way, linked to careful evaluation.

The NHS has chosen a pragmatic path through this by encouraging local innovation and national level support for scaling and spread. The NHS Challenge Prizes have provided funding, publicity and recognition to a wide range of fantastic ideas from across the country. For those already developing a specific innovation, the NHS Innovation Accelerator programme has provided the mentorship, knowledge, funding, and peer support to help 18 proven innovations to be adopted faster and more systemically through the NHS.

Another systematic challenge to the uptake and spread of an innovation is the resistance to change from the healthcare workers who will be impacted by it. We have previously treated new innovations, including digitisation of the healthcare record, as a simple 'technical' change. In reality, it is really an 'adaptive' change, which requires people themselves to adapt. ${ }^{17}$ Adopting an innovation is usually much more than just implementing a system - we need to understand the connection between people and technology, mobilise and engage staff to embrace change, and encourage them to reimagine the work itself.

The new NHS Clinical Entrepreneur's Programme is playing an important part of the push to encourage and nurture all clinical staff to get innovative ideas adopted within the NHS (Box 2). Now in its second year, with over 100 fellows across England, the NHS Clinical Entrepreneur's Programme provides regular educational 'pit stop' events, mentorship, funding and access to less-than-full-time training to pursue an innovative project. As this growing cohort develops the skills, knowledge and experience to innovate within the NHS, we hope to see many great innovations graduating from the programme and being adopted at scale across the system. ${ }^{18}$

\section{Conclusion}

As the largest single unified healthcare system on the planet, the NHS can appear a daunting place to innovate. However, that challenge is also an advantage. If you want to innovate at scale in healthcare, the NHS is the place.

In recent years, a vibrant healthcare start-up community and culture of innovation is beginning to form within the NHS, which offers the potential to revolutionise future patient care. We need to nurture this movement carefully, and work hard to address issues of regulation, intellectual property, funding, scalability and procurement. With appropriate focus, a culture of innovation will help the NHS to continue delivering worldclass universal healthcare to the population of the UK.

\section{Conflicts of interest}

The authors have no conflicts of interest to declare.

\section{References}

1 DeGusta M. Are smart phones spreading faster than any technology in human history? Cambridge, MA: MIT Technolology Review, 2012. www.technologyreview.com/s/427787/are-smart-phonesspreading-faster-than-any-technology-in-human-history/ [Accessed 10 April 2017].

2 National Audit Office. Financial sustainability of the NHS. London: NAO, 2016.

3 NHS England. Innovation into action: supporting delivery of the NHS Five Year Forward View. Redditch: NHS England, 2015.

4 NHS England. NHS Chief launches new fast track funding so NHS patients get treatment innovations faster. NHS England, 2016. www. england.nhs.uk/2016/06/treatment-innovations/ [Accessed 10 April 2017].

5 National Institute for Health Research. Invention for Innovation. www.nihr.ac.uk/funding-and-support/funding-for-research-studies/ how-to-apply/research-programmes/invention-for-innovation/ [Accessed 10 April 2017].

6 Lewis G. A network to support the new models of care with information governance. NHS England, 2016. www.england.nhs.uk/2016/10/ geraint-lewis-3/ [Accessed 10 April 2017].

7 Honeyman M, Dunn P, McKenna H. A digital NHS? An introduction to the digital agenda and plans for implementation. London: The King's Fund, 2016.

8 Department of Health, Hunt J. Jeremy Hunt challenges NHS to go paperless by 2018. London: Department of Health, 2013. www.gov. uk/government/news/jeremy-hunt-challenges-nhs-to-go-paperlessby-2018 [Accessed 10 April 2017]. 
9 NHS England. NHS Five Year Forward View. 2014. www.england. nhs.uk/ourwork/futurenhs/ [Accessed 10 April 2017].

10 Wachter RM. Making IT work: harnessing the power of health information technology to improve care in England. London: Department of Health, 2016.

11 Department of Health. The NHS as an innovative organisation: a framework and guidance on the management of intellectual property in the NHS. London: Department of Health, 2002.

12 Medicines and Healthcare products Regulatory Authority. Is your App a medical device? It's healthy to know: regulator issues updated guidance. London: MHRA, 2016. https://www.gov.uk/government/ news/is-your-app-a-medical-device-its-healthy-to-know-regulatorissues-updated-guidance [Accessed 10 April 2017].

13 US Department of Health and Human Services Food and Drug Administration. Mobile medical applications: guidance for industry and food and drug administration staff. 2015. www.fda. gov/downloads/MedicalDevices/DeviceRegulationandGuidance/ GuidanceDocuments/UCM263366.pdf [Accessed 10 April 2017].

14 Department of Health. Small and Medium-sized Enterprise (SME) Action Plan. London: Department of Health, 2012. www.gov.uk/ government/uploads/system/uploads/attachment_data/file/269987/ SME_Action_Plan.pdf [Accessed 10 April 2017].
15 NHS England. Health service embracing innovation as NHS England announces major trials to improve patient care. NHS England, 2016. www.england.nhs.uk/2016/01/embracing-innovation/ [Accessed 10 April 2017].

16 NHS England. NHS England announces $£ 101$ million of new funding for new care model vanguards. NHS England, 2016. www.england. nhs.uk/2016/12/vanguard-funding/ [Accessed 10 April 2017].

17 Heifetz RA, Grashow A, Linsky M. The practice of adaptive leadership: tools and tactics for changing your organization and the world. Boston: Harvard Business Press, 2009.

18 NHS England. The NHS Clinical Entrepreneur Programme. www. innovation.england.nhs.uk/clinical-entrepreneur [Accessed 10 April 2017].

Address for correspondence: Professor Tony Young, Postgraduate Medical Institute, Faculty of Medical Science, Anglia Ruskin University, Chelmsford Campus, Bishop Hall Lane, Chelmsford CM1 1SQ, UK.

Email: Tony.Young@anglia.ac.uk 